\title{
Una mirada desde el pensamiento complejo a la apropiación de las TIC en la docencia universitaria
}

\section{A look from complex thinking to the appropriation of ICT in university teaching}

Ing. Alejandra Colina Vargas MSc.

Universidad Tecnológica ECOTEC, Ecuador

Autor para correspondencia: acolina@ecotec.edu.ec

Fecha de recepción: 07 de Agosto de 2017 - Fecha de aceptación: 10 de Agosto de 2017

Resumen: El sistema educativo en la actualidad se encuentra asediado de profundos y significativos cambios generados por la incorporación de las Tecnologías de la Información y la Comunicación (TIC), lo cual obliga a asumir un gran reto y desafío de, en primer lugar, aprovechar las tecnologías con estrategias conscientes y profundas validando su contribución a los procesos educativos; y en segundo lugar, como respuestas a las demandas de una sociedad que, más que nunca, cambia a un ritmo acelerado. Una de las aristas de esa realidad, la viven en la actualidad la función docente pues se encuentra cercado por variadas y complejas expectativas, las cuales van desde la necesidad de formación básica e integral de las TIC para la vida ética y social, hasta la profesionalización especializada para la producción de conocimientos. Es por ello, que se desarrolla el presente artículo con el objetivo de analizar las distintas posiciones teóricas acerca de la "Apropiación de las TIC en la función docente universitario desde el pensamiento complejo", para comprender las vías y formas que las utilizan los docentes promoviendo y concienciando las mejoras en el aprendizaje de los estudiantes universitarios. El hilo conductor de la investigación se enfila hacia la mirada del pensamiento complejo como un método de construcción humano desde el punto de vista explicativo, tratando de comprender la realidad que vive el docente universitario a raíz del contexto ante descrito. Para ello, se consultaron diversas fuentes bibliográficas y documentales que explicitan las características del objeto de estudio desde diferentes perspectivas, lo que permitió asumir una postura teórica en cuanto al pensamiento complejo y la apropiación de las TIC. Se logró una sistematización de las definiciones de pensamiento complejo y la apropiación de las TIC sobre cómo aplicar al proceso formativo, especificándose en el docente universitario por ser un elemento de vital importancia que permita promover cambios en los estudiantes con vistas a la mejora del proceso educativo.

Palabras clave: Pensamiento Complejo; Apropiación; Tecnología; Docencia Universitaria

\begin{abstract}
The educational system is currently under siege of deep and significant changes generated by the incorporation of Information and Communication Technologies (ICT), which forces it to take on a great challenge and challenge of, firstly, taking advantage of technologies with deep and conscious strategies validating their contribution to educational processes; and secondly, as responses to the demands of a society that, more than ever, changes at an accelerated rate. One of the cornerstones of this reality is that the teaching profession is now surrounded by varied and complex expectations, ranging from the need for basic and integral training of ICT for ethical and social life, to professionalisation specialized for the production of knowledge. It is for this reason, that the present article is developed with the objective of analyzing the different theoretical positions on the "Appropriation of ICT in the university teaching function from the complex thinking", to understand the ways and forms that the teachers use them to promote and raising awareness of the learning outcomes of university students. The guiding thread of the research is directed towards the view of the complex thought as a method of human construction from the explanatory point of view, trying to understand the reality that the university teacher lives as a result of the context described above. For this, we consulted various bibliographic and documentary sources that explain the characteristics of the object of study from different perspectives, which allowed to assume a theoretical position regarding the complex thinking and the appropriation of the TIC. A systematization of the definitions of complex thinking and the appropriation of the ICTs on how to apply to the formative process was achieved, being specified in the university teacher because it is a vital element that allows to promote changes in the students with a view to the improvement of the educational process .
\end{abstract} Key Words: Complex Thinking; Appropriation; Technology; University Teaching 


\section{Introducción}

El aporte actual que han brindado las Tecnologías de Información y Comunicación (TIC), al desarrollo del conocimiento y la información ha sido indiscutible en diversos sectores, pues, por dar un ejemplo en el plano social, se resalta el apoyo que han dado a la democratización de la educación, o en lo económico, con una contribución al crecimiento de la economía mundial.

En este tenor, se concibe un nuevo modelo de crecimiento basado en la tecnología y el conocimiento, en la cual las instituciones se organizan para generar, adoptar, adaptar, difundir y usar los conocimientos, ya sean científicos, tecnológicos y gerenciales. De hecho, se pretende un desarrollo social y económico en función de los conocimientos de las personas a fin de crear valor e innovación que se traduzcan en productos y servicios, en definitiva bienestar para el ciudadano.

Las argumentaciones antes señaladas surgen de compaginar y evidenciar la coherencia entre la teoría con la práctica del docente universitario, las mismas parten del hecho de que en la actualidad están emergiendo profundos cambios y transformaciones socio-económicas, tecnológicas, políticas, culturales, así como también están surgiendo competencias requeridas para la comprensión de estos cambios, lo cual exige en la educación universitaria se estimulen las condiciones y la creatividad para propiciar una clara concepción sobre el futuro frente de cara a los retos del actual milenio.

Una de las aristas de esa realidad, la viven en la actualidad la función docente pues se encuentra asediada por variadas y complejas expectativas, las cuales van desde la necesidad de formación básica e integral de las TIC para la vida ética y social, hasta la profesionalización especializada para la producción de conocimientos.

Es por ello, que ante la dinámica y complejidad socio-histórica de esta era, las universidades ecuatorianas tienen un importante cometido en la búsqueda de respuestas para la solución de los problemas que enfrenta el país, a partir de las interrogantes que plantea la búsqueda del conocimiento, dada su mutable y evasiva esencia, para que desde allí se asuma una actitud antropoética de simbiosis planetaria. Al respecto, Morin (2002), plantea que dentro de las alternativas de respuesta a las dificultades antes mencionada se han dado reformas en cuanto a la flexibilidad, a la aligeración, a las instalaciones, pero estos cambios o transformaciones, cuando lo único que se hace, son "pequeñas reformas" que ocultan aún más la necesidad de la reforma de pensamiento.

De allí, que la propuesta del artículo, se enfoca hacia la mirada del pensamiento complejo como un método de construcción humano desde el punto de vista explicativo, tal como lo refiere (Tobón, 2005) interpretando y comprendiendo el mundo y el ser humano, a partir de una serie de partes y elementos que se relacionan, organizan y complementa diferentes factores de la naturaleza.

A partir de esa significancia se desea comprender el contexto institucional de las distintas Universidades Ecuatorianas, a fin de construir una visión teórica que sustente la apropiación de las TIC en la función docente del profesorado universitario que surge desde el pensamiento complejo, para lo cual se presentan las interrogantes complementarias que orientan esta investigación, las cuales se entretejen en ¿Cuál es la epistemología del pensamiento complejo desde la perspectiva de la función pedagógica del docente universitario? ¿Cuál es la visión teórica de la apropiación en TIC de los docentes en las carreras de Ingeniería de Sistemas de las Universidades de Guayaquil en el Ecuador" desde el marco conceptual del pensamiento complejo?

\section{Metodología}

La investigación se basó en un análisis cualitativo de información y datos relacionados a la problemática. Comparando para ello distintas fuentes de información recopiladas de algunos artículos o documentos de trabajo científicos realizados por los miembros de grupos que realizan proyectos 
relacionados con la educación y la integración de las TIC en el aula. Para ello, se tomó como eje de análisis la identificación de la función del profesor y a partir de ella, aportar algunas ideas que apuntan a las competencias docentes que necesitarán para apropiarse de las TIC, con miras comprender el desempeño docente en esta época de transiciones, tanto en la forma de resignificar su concepto de la educación y el aprendizaje, como en la conducción de la formación profesional de los estudiantes.

\section{Profesorado Universitario}

El profesorado universitario es un tipo de profesional que realiza un servicio a la sociedad a través de la universidad. Se caracteriza por ser reflexivo, critico, competente en el ámbito de su disciplina, capacitado para ejercer la docencia y para realizar actividades de investigación.

De igual manera, se espera de estos profesionales que el conocimiento impartido en aulas, talleres o laboratorios sea relevante para la formación teórica y práctica de los estudiantes, éstos últimos para que sean cada vez más autónomos, avancen en sus procesos de estudios y en la interpretación crítica del conocimiento y de la sociedad a la vez que adquieren una capacitación profesional.

De igual manera, se identifican a los profesionales de la educación superior de acuerdo al Consejo de Educación Superior - CES (2016) en el Reglamento de Carrera y Escalafón del Profesor e Investigador del Sistema de Educación Superior con tres actividades o funciones: de docencia, de investigación, de vinculación con la sociedad y de dirección o gestión académica. En virtud de ello, se define al profesor como todo aquel que enseña de una manera organizada, en virtud de la preparación académica adquirida, que acredita a través de algún título, en alguno de los niveles del sistema escolar o estudio reglado.

\section{Roles o funciones del profesor universitario.}

Las funciones que el profesor universitario asume en su praxis docente son dinámicas y se han ido adaptando a los cambios de la sociedad, puesto que se han replanteado en algunos casos nuevos objetivos, estructuras, medios a nivel curricular y pedagógico. Todo este escenario descrito trae consigo hoy en día la demanda un nuevo rol de profesor cuya principal función sea la de ser formador de futuros profesionales con diferencias claras entre sí: en proyectos de formación, desarrollo del currículo, determinación de competencias, habilidades y destrezas para el cometido de una función laboral futura.

\section{Competencias del docente universitario.}

Antes de dar comienzo a esta sesión es conveniente hacer referencia a la existencia de una diversidad de acepciones acuñadas al término de competencias para luego destacar el significado de competencias del docente universitario. Comienzo con, Vasco (2003) quien resalta el término como la capacidad y abordaje de tareas nuevas, definiéndola entonces a la "capacidad para el desempeño de tareas relativamente nuevas, en el sentido de que son distintas a las tareas de rutina que se hicieron en clase o que se plantean en contextos distintos de aquellos en los que se enseñaron”.

Tobón (2005) define una postura en torno al vocablo de competencia, como aquellos procesos complejos de desempeño ideales en determinados contextos haciendo uso para ello de diferentes saberes (saber ser, saber hacer, saber conocer y saber convivir), los cuales permitirán la realización de actividades o resolución de problemas, asumiendo esa acción como reto, motivación, flexibilidad, creatividad, comprensión y emprendimiento, dentro de una perspectiva de procesamiento metacognitivo de mejoramiento continuo y compromiso ético. Contribuyendo al final con el desarrollo personal, afianzando el tejido social, la búsqueda continua del desarrollo económico-empresarial sostenible, así como también el cuidado, protección del ambiente y de las especies vivas. 
En esta última definición Tobón, destaca elementos significativos del término competencia, pues permite dilucidar aquellos aspectos a focalizar en el marco del presente estudio investigativo, no como una representación ideal o modelo del proceso educativo universitarios sino centrado únicamente en aquellos aspectos conceptuales y metodológicos de la educación y la gestión del talento humano que viabilicen la docencia universitaria en el contexto complejo de la sociedad actual.

En este mismo orden de, Zabalza (2003) expresa que las competencias profesionales docentes del profesorado universitario estarán conformadas por un conjunto de conocimientos, saber hacer, habilidades y aptitudes que permitirá a los profesionales desempeñar y desarrollar roles de trabajo en los niveles requeridos para el empleo.

Haciendo alusión a las distintas competencias que se debería considerar dentro del sistema formativo universitario, Zabalza señala:

a) Planificar el proceso de enseñanza-aprendizaje

b) Seleccionar y preparar los contenidos disciplinares

c) Ofrecer información y explicaciones comprensibles y bien organizadas (competencia comunicativa)

d) Manejo de las nuevas tecnologías

e) Diseño la metodología y organizar las actividades.

f) Organización de los espacio

g) Selección del método de trabajo

h) Selección y desarrollo de las tareas instructivas

i) Comunicarse-relacionarse con los alumnos

j) Tutorizar al estudiantado

k) Evaluar los aprendizajes.

1) Reflexionar e investigar sobre la enseñanza

m) Identificarse con la institución y trabajar en equipo.

A partir de las definiciones antes presentada y dada la importancia para el tema objeto de estudio se plantea la existencia de nuevas competencias que debe asumir el docente universitario en el contexto de la apropiación de las tecnologías, centrando su accionar en actividades de tutorización y guía del proceso, diseñador de experiencias y contenidos, entre otros, así como la consolidación de espacio de análisis, discusión y construcción de nuevas maneras de la producción de conocimiento.

\section{Función o Actividad Docente.}

La función docente no consiste únicamente en la exposición de conocimientos de una ciencia, sino en cómo tal la ciencia produce su conocimiento, cuáles son los medios conceptuales y metodológicos para producir tales conocimientos, por qué se producen tales conocimientos en ruptura o desarrollo respecto a otros conocimientos.

En otro tenor, Sánchez-Parga (2003) expresa que la función docente más que una enseñanza los conocimientos de una ciencia enseña a pensar una determinada ciencia y a pensar científicamente sus conocimientos. La función docente del profesorado universitario responde al por qué de los conocimientos, a su razón y a la lógica que los explica.

Siendo entonces necesario caracterizar a la profesionalidad en la docencia con lo siguiente:

1. Delimitación de un ámbito específico de actuación.

2. Preparación técnica y científica para resolver los problemas propios de su ámbito de actuación.

3. Compromiso de actualización y perfeccionamiento de los conocimientos y habilidades que le son propios. 
4. Unos ciertos derechos sociales como individuo y como colectivo profesional.

5. Autonomía de actuación.

6. Compromiso deontológico con la práctica docente.

En este sentido, las actividades de docencia para las universidades e institutos politécnicos comprenden una serie de actividades según el CES (2016) en el Reglamento de Carrera y Escalafón del Profesor e Investigador del Sistema de Educación Superior, establece:

- Impartición de clases presenciales, virtuales o en línea, de carácter teórico o práctico en la institución o fuera de ella, bajo responsabilidad y dirección de la misma

- Preparación y actualización de clases, seminarios, talleres, entre otros;

- Diseño y elaboración de libros, material didáctico, guías docentes o syllabus;

- Orientación y acompañamiento a través de tutorías presenciales o virtuales, individuales o grupales;

- Visitas de campo y docencia en servicio;

- Dirección, seguimiento y evaluación de prácticas y pasantías profesionales;

- Preparación, elaboración, aplicación y calificación de exámenes, trabajos y prácticas.

- Dirección y tutoría de trabajos para la obtención del título, con excepción de tesis doctorales o maestrías de investigación.

- Dirección y participación de proyectos de experimentación e innovación docente.

- Diseño e impartición de cursos de educación continua o de capacitación y actualización.

\section{La función docente universitario en la Sociedad Actual.}

En la sociedad actual, la acción de enseñar está sometida a constantes cambios, los cuales sobrevienen a velocidad de vértigo, convirtiéndola en una profesión difícil e incómoda que se desarrolla en un marco de incertidumbre y de profundas innovaciones tecnológicas. Estos cambios se manifiestan tanto en su práctica y en la organización de la enseñanza, así como para el desarrollo de competencias claves en la formación universitaria moderna, tales como la innovación, el trabajo en equipo y una visión global y multicultural.

Ahora bien, todo el escenario presentado esboza numerosos retos a la enseñanza, pues se requiere la formación de personas más preparadas, con mayores capacidades para aprender y mayores posibilidades de desarrollo personal y profesional. Es decir, se requieren personas que sean capaces de colaborar más y mejor en la construcción de una sociedad más justa, que elimine la desigualdad y la exclusión respetando la diversidad y a la identidad de cada persona.

Debido a ello se están produciendo en la sociedad actual la demanda de redefinir la función docente, pues los roles que tradicionalmente ha desempeñado el docente de manera cautelosa haciendo uso de curriculum conservadores, resultan hoy inadecuados. Pues, a los jóvenes universitarios actualmente les llega la información por múltiples vías: la televisión, la radio, el computador, el internet, entre otros, mientras que ellos quedan indefensos. Esto se debe a que poseen una habilidad natural para apropiarse de las TIC e incorporarlas a sus actividades escolares y de socialización, hecho que se hace evidente en la manera en que utilizan los medios digitales para comunicarse e interactuar.

En este marco de ideas, las universidades como instituciones dinámicas que establecen vínculos con la sociedad contemporánea, ha de hacer frente a los cambios demandados por la incorporación de las Tecnologías de Información y Comunicación (TIC). Por lo tanto, están obligadas de acuerdo a lo que señala Salinas (2004) a:

"revisar sus referentes actuales y promover experiencias innovadoras en los procesos de enseñanza aprendizaje, apoyándose en las TIC y haciendo énfasis en la docencia, en los cambios de estrategias 
didácticas de los profesores y en los sistemas de comunicación y distribución de los materiales de aprendizaje; es decir, en los procesos de innovación docente, en lugar de enfatizar la disponibilidad y las potencialidades de las tecnologías".

Cabe considerar, que las TIC pueden permitir según afirmaciones de Cebrián (2003) la posibilidad de ayudar a que la enseñanza esté basada en el aprendizaje, centrada en el alumno, en un aprendizaje flexible, abierto y activo por parte del mismo. Estas tecnologías, generalmente, pueden ser utilizadas para cubrir las carencias de la enseñanza presencial y ser un complemento para el aprendizaje, permitiendo un amplio abanico de estilos de enseñanza.

Por su parte, en el informe elaborado por la Organización de las Naciones Unidas para la Educación, la Ciencia y la Cultura coordinado por Delors (1996) se plantea como urgente que en las instituciones de educación superior se dé "una notable transformación de las funciones del profesorado a requerimiento de la sociedad de la información". Sugiriendo en ese documento, que deben ser los propios profesores los que racionalicen y estructuren las nuevas formas de conocimiento a través de las nuevas tecnologías; esto no significa que debe disminuir su papel, sino que, al contrario, debe constituir para ellos una fuente de innovación metodológica y de profusión y enriquecimiento de sus actividades docentes.

Teniendo en cuenta que, Salinas (2004) esboza que el docente en este contexto deja de ser fuente de todo conocimiento y pasa a actuar como guía de los alumnos, facilitándoles el uso de los recursos y las herramientas que necesitan para explorar y elaborar nuevos conocimientos y destrezas; pasa a actuar como gestor del conjunto de recursos de aprendizaje y a acentuar su papel de orientador y mediador.

Partiendo de ello, se requiere que los docentes sean especialistas en sus materias, y tengan vocación pedagógica. Es decir, que no sólo sepan lo que enseña sino sobre todo fundamente lo que sabe; en otras palabras, se requiere que le dé sentido a lo que se enseña contextualizando a nivel general y del de la sociedad que lo acoja.

Dentro de ese marco de ideas, la función del docente hoy, se ha ubicado dentro de un enfoque de enseñanza centrado en el alumno, lo cual trae consigo que deben ser atendidas aquellas actitudes, políticas y prácticas que pueden ampliar o disminuir la distancia de los alumnos. De igual manera, en ese proceso de enseñanza-aprendizaje resalta el hecho de promover en el alumno el crecimiento personal, enfatizando la facilitación del aprendizaje antes que la transmisión de información, y fomentando el aprendizaje autónomo, caracterizado tanto por la participación como por la anticipación.

Se puede considerar entonces que, a partir de esta realidad las instituciones de educación universitaria exigen que su personal docente deba poseer ciertos conocimientos, habilidades y actitudes para ejercer en el aula una práctica educativa innovadora. En tal sentido, Cebrián (2003) señala que los procesos de cambios sociales, tecnológicos y científicos demanda una formación inicial y continua del profesorado, donde el dominio de las TIC es fundamental para lograr asumir esos nuevos roles por parte de ese personal.

\section{Apropiación Social}

La apropiación puede definirse como tomar algo que pertenece a otros y hacerlo propio, esto implicaría el traslado de la responsabilidad de transferir el conocimiento adquirido del individuo a otros contextos (Colás y Jiménez, 2008). El concepto se deriva de un modelo social de aprendizaje en el que se asocian términos como el dominio, la internalización y el privilegio del conocimiento. 
Es por ello, que a través de este proceso de apropiación de la tecnología se establece de acuerdo a los autores antes mencionados, la transformación simultánea al usuario y a la tecnología; es decir, no sólo da lugar a que el usuario cambie en sus conocimientos y sus habilidades, sino que también el conocimiento adquirido del individuo a otros contextos

La apropiación de una tecnología es un proceso que, simultáneamente, transforma al usuario y a la tecnología; es decir, no sólo da lugar a que el usuario cambie en sus conocimientos y sus habilidades, sino que también causa transformaciones en las propiedades de la tecnología (Overdijk y Diggelen, 2006). Por otra parte, Colás, Rodríguez y Jiménez (2005) mencionan que la apropiación plantea la manera en que las tecnologías son asumidas por los sujetos, estructurando sus formas de interpretar la realidad y constituyendo la base de su aprendizaje. Otros autores también describen este concepto como el núcleo de la relación entre sujeto y mundo particulares, refiriéndose al proceso en el que cada sujeto en particular utiliza los sistemas de usos y expectativas en relación con su interés y concepciones (Gutiérrez y Quiroz, 2007).

El modelo de evaluación apropiación tecnológica según Orozco y Sánchez plantea tres criterios de referencia: conocimiento, utilización y transformación. Entre la categoría de conocimiento de la tecnología comprende la representación que los docentes tienen de la misma y de sus usos; puede ir desde un nivel descriptivo hasta la generalización a múltiples escenarios; la utilización representa el empleo común de prácticas educativas que involucran apropiación de las tecnologías de información y comunicación (TIC), mientras que la tercera categoría, llamada de transformación, se relaciona con la modificación o adaptación que realizan los profesores en las prácticas que involucran el uso de la tecnología en el salón de clase.

El término de apropiación tecnológica se puede relacionar con los cambios que se producen en los profesores como consecuencia del empleo de la tecnología en sus cursos; en este sentido el trabajo docente se convierte en algo diferente cuando se le incorpora el uso de tecnología de información, ya sea de manera voluntaria o forzada, porque se integran nuevos conocimientos y habilidades que se manifiestan en el grado de dominio y apropiación que presenta el profesor (McAnally-Salas, Navarro y Rodríguez, 2006).

\section{Pensamiento complejo}

\section{Caracterizando el pensamiento complejo de Morín.}

En la sociedad contemporánea surge la complejidad como una perspectiva novedosa y marginal en la ciencia; su carácter de novedad radica en que implica un quiebre o discontinuidad en la historia de la ciencia, es decir en la racionalidad científica occidental. La complejidad introduce, según Rodríguez y Aguirre (2011) en el terreno de las ciencias, contempla una racionalidad post-clásica que habilita e incorpora problemas ignorados o vedados por el pensamiento científico moderno, los cuales involucran, cuestiones relativas al desorden, el caos, la no-linealidad, el no-equilibro, la indecibilidad, la incertidumbre, la contradicción, el azar, la temporalidad, la emergencia, la auto-organización.

De la complejidad existen de acuerdo a lo que establece Maldonado (1999) existen varias visiones como forma de racionalidad, varias formas de comprenderla, varios planos. Uno de ellos es el de o hacia la complejidad como método. Siendo muy popular debido no solamente al lenguaje mismo que emplea, sino a la introducción de numerosos neologismos y grafismos (como flechas, guiones intermedios, backslashes), es elemental y directo. Siendo Edgar Morin su precursor.

El filósofo Morín introduce en su obra denominada el Método Volumen I, la necesidad de concebir o de desarrollar un nuevo método, el cual sea diferente al de la ciencia clásica, denominado como un anti - método. El método propuesto consiste en el aprendizaje del pensamiento relacional, no 
como simplemente un pensamiento, sino, más considerablemente, una actitud general hacia el mundo, la naturaleza, la vida, en fin, también hacia el propio conocimiento.

Evidentemente, el mérito central del pensamiento de Morín tal como lo expresa Maldonado (Ob. cit) radica en lo que se llama "pensamiento relacional". Este pensamiento se caracteriza por el uso claramente exagerado de figuras y de grafismos conjuntamente con el énfasis enorme en la configuración de nombres y conceptos fundados en prefijos en la obra de Morín, entre los que se puede mencionar Pre / eco / geno / feno / trans / epi / multi / retro / peri / entre otros, lo cual denota el esfuerzo por desarrollar y enseñar un pensamiento relacional; o pensamiento de redes.

Por otro lado, otro de los aspectos que destaca el pensamiento complejo de Morín, es el esfuerzo por recuperar o por situar lo que él denomina lo vivo del sujeto (Le vif du sujet), esto es; la centralidad absoluta del sujeto cognoscente. Desde este punto de vista, la obra de Morín se inscribe perfectamente dentro de la más clásica tradición francesa, a saber, es una filosofía del sujeto y, bien entidad, al límite, puede identificarse con una filosofía de la conciencia.

En otro orden de idea, Morín (Ob cit.) define los sistemas complejos siguiendo un abordaje nolineal de la realidad y se afirmó, entre otros, en los siguientes enunciados:

un sistema complejo no puede ser analizado, en principio, en forma fragmentaria, es decir, por partes; se halla constituido por un sistema de elementos que tiene un/múltiples sentido/s en la intimidad del sistema considerados en un horizonte temporal limitado y puede sufrir transformaciones y cambios bruscos; se diferenció de un sistema complicado, dado el hecho de que la dificultad de predicción no se encontró en la incapacidad del observador de tener en cuenta todas las variables que influyeron su dinámica, sino en la "sensibilidad" del sistema a las condiciones iniciales (debemos tener en cuenta que las condiciones iniciales diferentes condujeron a evoluciones extremadamente diversas) a las cuales se agregaron los efectos de los procesos de auto organización (procesos que se hallaron condicionados por las interacciones de los subsistemas componentes, y que tuvieron como efecto la aparición espontánea y no predecible de unas determinadas relaciones de orden); un sistema complejo incorporó una evolución que no resultó del análisis de sus respuestas a un estímulo dado (bajo la forma de un análisis dinámico), sino que su dinámica y su evolución necesitaron de abordajes específicos y distintos.

Morín explica en su libro El método I (1993), que:

Un sistema es una interrelación de elementos que constituyen una entidad global o unidad global. Tal definición comporta dos caracteres principales: el primero es la interrelación de los elementos y el segundo es la unidad global constituida por estos elementos en interacción (...) se puede concebir el sistema como unidad global organizada de interrelaciones entre elementos, acciones o individuos (pp. 123-124).

El paradigma de la complejidad establece un marco conceptual que permite establecer interrelaciones e intercomunicaciones reales entre las diversas disciplinas, y provocó un fecundo diálogo entre especialistas, metodologías y lenguajes específicos.

La complejidad por su parte apareció, justamente, como resultado de la organización del todo bajo la presión de las infinitas combinaciones de simultáneas, y que abundaron en interrelaciones no-lineales. La complejidad nació de la interacción de las partes que lo componen, es decir, la complejidad se manifestó en el sistema mismo.

En el siguiente acápite se describen los principios del pensamiento complejo de Morin, los cuales sirven de sustento en el objeto de estudio. 


\section{Principios del Pensamiento Complejo de Morín.}

El pensamiento complejo como nueva forma de pensar hace uso de la abstracción a fin de que sus producciones de conocimiento se cimenten por referencia obligada a un contexto (cerebral, social, espiritual). El pensamiento complejo tal como lo plantea Morín (1981) busca que el conocimiento se integre y globalice religando las partes al todo, el todo a las partes y las partes entre sí, sin embargo teniendo conciencia de que es imposible conocer el todo: Es necesario movilizar el todo, pero es imposible conocer todo el mundo.

Este pensamiento es considerado por Gómez y Jiménez (2002) como un modo de pensar que intenta asumir el desafío, que le proponen la incertidumbre y la contradicción. Para ello es necesario un cambio de paradigma que relativice y ponga en cuestión los principios de conocimiento en que se funda el pensamiento clásico. Es decir, el pensamiento complejo debe complementar y confrontar el modo de pensar que separa con un modo de pensar apoyado en unos principios de conocimiento tales que devenga capaz de concebir la organización, que religue, contextualice y globalice. Pero el pensamiento complejo, al mismo tiempo que lucha por conectar lo separado, debe ser capaz de reconocer lo anormal, lo singular, lo concreto.

Para la construcción de este nuevo conocimiento es necesario asumir el problema epistemológico, es decir no como una epistemología de segundo orden sino un saber del conocimiento del conocimiento, como la aventura del método de la complejidad. Como todo modo de pensar, el pensamiento complejo tiene que establecer distinciones, trabajar con categorías de análisis. La categoría compleja de organización es una de las herramientas vitales del pensamiento complejo: la noción de organización trae a su campo semántico las nociones de orden, desorden y sistema.

\section{El principio dialógico o de dialogización}

En este contexto, el principio dialógico se define como un principio de conocimiento que une o pone en relación ideas o principios de dos lógicas que de suyo son antagónicas. Esto es, él une dos principios o ideas que se excluyen mutuamente, pero que son inseparables dentro de una misma realidad o fenómeno.

El principio dialógico concede al pensamiento en sus asociaciones y conexiones de conceptos o enunciados que se contradicen el uno al otro, pero que deben aparecer como dimensiones articuladas de lo mismo. Su vocación epistemológica es captar el modo de existencia, el funcionamiento y las interdependencias contextuales de un "fenómeno" complejo. El principio dialógico es un principio de complejidad en el sentido de que afina el pensamiento para captar las contradicciones fecundadas que aparecen cada vez que tiene que vérselas con un sistema complejo,con la dimensión generativa de su organización. Así, para poder describir la dinámica de un sistema complejo es vital concebir una dialógica, un diálogo de lógicas entre orden, desorden y organización.

El principio dialógico conduce a la idea de "unidualidad compleja". La unidualidad entre dos términos significa que éstos son, a la vez, ineliminables e irreductibles. Por separado, cada término o cada lógica resultan insuficientes, por lo que hay que relacionarlos a ambos y hacerlo en forma de bucle. Ninguno de los dos términos es reducible al otro ( $\mathrm{y}$ en este sentido hay dualidad), pero tampoco son nítidamente separables, pues confluyen mutuamente (y en este sentido son uno).

En los problemas se suele ir en contra con tesis antagonistas que se plantean como enfrentadas, irreconciliables y excluyentes. Este modo de plantearlas es resultado del pensamiento simplificador, disyuntor y reductor que subyace a ambas tesis. Un paradigma de la complejidad posibilita la asociación de las tesis o proposiciones contradictorias. Consideradas juntamente, las tesis alternativas suelen expresar verdades. Pero, al rechazar la tesis contraria y, consiguientemente, la parte de verdad que ésta contiene, aisladamente cada tesis resulta insuficiente y mutilante. Un paradigma de la complejidad nos insta a ver e integrar las dos tesis antagonistas, a desarrollar una visión poliocular. 


\section{El principio de recursión}

En términos de complejidad, la noción de recursividad está asociada a la idea de bucle retroactivo, pero lo supera largamente; por tanto, va más allá de la idea cibernética de regulación. El principio de recursividad conduce al pensamiento complejo a las ideas de autoproducción y auto organización. Estas dos ideas, junto con el principio de recursividad, sirven para la comprensión científica de los sistemas complejos: la vida, el universo, la sociedad, etc.

El principio de recursividad es, pues, un principio de pensamiento fundamental no solo para asir la retroacción de los productos sobre el productor, sino también para reconocer y traducir, en términos de la teoría, aquellas entidades y características que son productos a la vez que productores y causas del mismo proceso que las produce: esto es un bucle recursivo.

El principio de recursividad es, por tanto, un principio vital a la hora de pensar la organización de un sistema complejo.

\section{El principio hologramático} totaliza.

La voz griega holon significa "todo". Pero no se trata de una totalidad. Es un todo que no El principio hologramático nos guía y nos permite concebir una de las características más sorprendentes e importantes de las organizaciones complejas: En una organización, el todo está inscrito en cada una de sus partes. Se trata, obviamente, de una inscripción estructural del todo en la parte. Se da bajo tres modalidades o maneras de estar el todo en las partes: Holonómica, Hologramática y Holoscópica. (Ver Tabla No 1.)

En este sentido, el holograma parece capturar, siquiera de forma metafórica, un principio de organización general que estaría presente en muy diversos dominios de lo real: cada parte contiene dentro de sí el todo; cada parte debe su singularidad justamente a que, controlada por la organización del todo (producido por las interacciones de las partes), una pequeña parte del todo se expresa en él, pero, al mismo tiempo, sigue siendo portadora de las virtualidades del todo.

De lo anterior deriva, que el pensamiento complejo dispone de la posibilidad de religar el todo con la parte y la parte con el todo, así como de la posibilidad de no recaer en las engaños de la simplificación.

\section{Tabla No. 1. Modalidades del Principio Hologramático}

\begin{tabular}{|c|c|c|}
\hline Modalidad & Definición & Ejemplo \\
\hline Holonómica & $\begin{array}{l}\text { El todo, en tanto que todo, puede gobernar } \\
\text { las actividades locales. }\end{array}$ & $\begin{array}{l}\text { El cerebro, en tanto que todo, gobierna los núcleos } \\
\text { de neuronas que lo gobiernan. }\end{array}$ \\
\hline Hologramática & $\begin{array}{l}\text { El todo puede, aproximadamente, estar } \\
\text { inscrito o engramado en la parte inscrita en } \\
\text { el todo. }\end{array}$ & $\begin{array}{l}\text { En cada célula está la totalidad de la información } \\
\text { genética del organismo. }\end{array}$ \\
\hline Holoscópica & $\begin{array}{l}\text { El todo puede estar contenido en una } \\
\text { representación parcial de un fenómeno o de } \\
\text { una situación. }\end{array}$ & $\begin{array}{l}\text { Es lo que ocurre en los procesos de rememoración y } \\
\text { de percepción. }\end{array}$ \\
\hline
\end{tabular}

Fuente: Adaptación de Gómez y Jiménez (2002)

\section{Conclusiones}

Este profesional se distinguirá por ser una persona reflexiva, crítica, competente en el ámbito de la propia disciplina, y deberá desempeñar tres funciones: Docencia, Investigación y Gestión Académica. Cabe destacar que, en este informe únicamente se abordara la función de docencia o enseñanza, siendo está considerada por Bartha (2002) como un proceso organizado, intencional y sistemático que orienta los procesos de enseñanza-aprendizaje, exigiendo el dominio de la especialidad, así como el conocimiento de las estrategias, técnicas y recursos psicopedagógicos que promuevan la interacción didáctica y los aprendizajes significativos en los estudiantes. 
En dicho proceso, la tarea del profesor girar en torno a la promoción en el participante de captar conceptos sobre la profesión y la cultura, a la par del desarrollo de habilidades y el reforzamiento de determinadas actitudes que lo impulsen a ejercer de la mejor manera su vocación profesional y humana; siendo eje central el crecimiento del estudiante, no sólo en el tema que se imparte, sino en su desarrollo como individuo.

Bajo ese hilo conductor, la docencia universitaria resulta ser una tarea distinta a otras y con complejidad notable, sobre todo en esta época donde pasar de estar centrada en la enseñanza a otra que esté centrada en el aprendizaje de los estudiantes. A partir de este nuevo enfoque González (2007) hace énfasis en que "los docentes tendrán que actuar como guías, facilitadores, mediadores de proceso, modelos, investigadores, y punto referencia en el proceso de aprendizaje". Estableciendo, un nuevo rol dentro del desempeño docente, de manejador de perspectiva globalizada del conocimiento al de asumirá la función de promotor, animador, incorporador de contenidos y tutor, entre otras.

Desde esta perspectiva, Zabalza (2003) plantea que el desempeño docente es complejo pues en él se suscitan situaciones incontrolables e impredecibles, llenas de incertidumbre y de azar; hay que estar pendientes de cada uno de ellos, atender sus individualidades; supervisar el proceso que va siguiendo; facilitar su progreso a través de los recursos didácticos.

Es a partir de este contexto, que Morin (1999) plantea una reforma del pensamiento y por tanto una reforma de la enseñanza. Siendo así, que en su obra "La Cabeza bien puesta" aboga por la misión de la enseñanza centrado en trasmitir, no saber puro, sino una cultura que permita comprender nuestra condición y ayudarnos a vivir.

De igual manera, ese autor hace alusión a la problemática de enseñanza partiendo de las contradicciones y a la resonancia de las acciones del ser humano, postulando tres ideas como son: contextualizar, globalizar y anticipar. Se quiere con ello significar, la generación de una forma de pensamiento de la realidad humana que no aísle sino que contextualice, que no vea la localmente sino global y por último que no se centre en el aquí y el ahora sino que anticipe los efectos de cualquier acción. Todo ello con el propósito de permitir un conocimiento pertinente, y no mutilado, y que al final se desarrolle un pensamiento centrado en la condición humana del mundo y no únicamente pedazos de ese pensamiento.

La idea es que entro del desempeño docente a raíz de los cambios propuestos por la sociedad moderna, destaca el hecho de que estos profesionales no acumulan conocimientos sino investigan, piensan, se comunican, tienen iniciativa, aprenden a trabajar por su propia cuenta y de esta manera contribuyen al desarrollo comunitario

Se define en este sentido, la firme convicción de que el docente rompa con la concepción de ser simple transmisión del saber y que participe activamente en la investigación de su propia práctica y en la resolución de los problemas que le plantea su entorno social y que contribuya con la transformación del sistema educativo. Así como también, pone en evidencia a necesidad de un conocimiento multidisciplinario, pues la educación, tradicionalmente, ha formado al ser humano con esquemas disyuntores, que buscan la comprensión a través de la separación de las partes.

\section{Bibliografía}

Cebrian, M. (2003). Enseñanza Virtual Para La Innovación Universitaria. Narcea, S.A. Ediciones. España Colás, P. Y Jiménez, R. (2008). "Evaluación Del Impacto De La Formación (Online) En Tic En El Profesorado. Una Perspectiva Sociocultural", Revista De Educación, 346, Mayo-Agosto, Pp. 187215. Disponible En: Http://Www.Revistaeducacion.Mec.Es/Re346/Re346_07.Pdf

Colás, P.; Rodríguez, M. Y Jiménez, R. (2005). "Evaluación De E-Learning. Indicadores De Calidad Desde El Enfoque Sociocultural”, Revista Electrónica Teoría De La Educación Y Cultura En La Sociedad De La Información Monográfico: Estado Actual De Los Sistemas E-Learning, Disponible: Http://Www.Usal.Es/ Teoriaeducacion/Rev_Numero_06_2/N6_02_Art_Colas_Ro

Driguez_Jimenez.Ht)

Consejo De Educación Superior (2016). Reglamento De Carrera Y Escalafón Del Profesor E Investigador Del Sistema De Sucación 
Http://Www.Ces.Gob.Ec/Doc/Gaceta_Ces/Reglamento/Reglamento\%20de\%20carrera\%20y\%20e scalafn\%20del\%20profesor\%20e\%20investigador\%20del\%20sistema\%20de\%20educacin $\% 20$ su perior-Codificada.Pdf

Delors, J. (1998). La Educación Encierra Un Tesoro. Madrid: Santillana-Unesco.

Gómez M., R. Y Jiménez, J. (2002) Manual De Iniciación Pedagógica Al Pensamiento Complejo. De Los Principios Del Pensamiento Complejo. Instituto Colombiano De Fomento De La Educación Superior. Unesco

González, N. (2007). Formación Docente Centrada En Investigación. Una Propuesta Interactiva Para Construir Aprendizajes. Colección Textos Universitarios. Universidad Del Zulia, Venezuela.

Gutiérrez, E. Y Quiroz, R. (2007). "Usos Y Formas De Apropiación Del Video En Una Secundaria Incorporada Al Proyecto Sec Xxi". Revista Mexicana De Investigación Educativa, 32(12), Pp. 337 258.

Maldonado, C. (1999). Visiones Sobre La Complejidad, 2a Edición, Colección "Filosofía Y Ciencia" No. 1, Santafé De Bogotá, Editor, Y Coautor. Disponible: Http://Carlosmaldonado.Org/Articulos/Esbozo\%20dr.\%20maldonado.Pdf

Mcanally-Salas, L.; Navarro H., M.; Rodríguez L., J.; (2006). La Integración De La Tecnología Educativa Como Alternativa Para Ampliar La Cobertura En La Educación Superior. Revista Mexicana De Investigación Educativa, Enero-Marzo, 11-30. Disponible: Http://Www.Redalyc.Org/Pdf/140/14002803.Pdf

Morin, E. (1993). El Método I: La Naturaleza De La Naturaleza. Madrid: Cátedra.

Morin, E. (1998). Introducción Al Pensamiento Complejo. Editorial Gedisa. España (1999). La Cabeza Bien Puesta. Buenos Aires: Nueva Visión.

Morin,E.(1999). La Cabeza Bien Puesta. Repensar La Reforma,Reformar El Pensamiento.Bases Par a Una Reforma Educativa. Buenos Aires: Nueva Visión.

Overdijk, M. Y Diggelen, V. D. (2006). "Technology Appropriation in Face-To-Face Collaborative Learning", First European Conference on Technology Enhanced Learning, 1-2 De Octubre, Creta, Grecia: Ceur-Ws. Disponible En: Http://Ftp.Informatik.Rwth-Aachen.De/Publications/CeurWs/Vol-213/Paper17.Pdf

Rodríguez Z., L Y Aguirre, J. (2011). Teorías De La Complejidad Y Ciencias Sociales Nuevas Estrategias Epistemológicas Y Metodológicas. Nómadas. Revista Crítica De Ciencias Sociales Y Jurídicas. Disponible: Https://Pendientedemigracion.Ucm.Es/Info/Nomadas/30/Rdzzoya_Aguirre.Pdf

Salinas, J. (2004). "Innovación Docente Y Uso De Las Tic En La Enseñanza Universitaria". Revista De Universidad Y Sociedad Del Conocimiento (Rusc). Uoc. Vol. 1, $\mathrm{N}^{\circ}$ 1. Artículo En Línea. Disponible: Http://Www.Uoc.Edu/Rusc/Dt/Esp/Salinas1104.Pdf

Sánchez-Parga, J. (2003). La Docencia Universitaria. Para Un Manifiesto Antipedagógico. Universidad Politécnica Salesiana.

Tobón, S. (2005). Formación Basada En Competencias: Pensamiento Complejo, Diseño Curricular Y Didáctica. Bogotá: Eco Ediciones.

Vasco, C.E. (2003). Objetivos Específicos, Indicadores De Logros Y Competencias ¿Y Ahora Estándares? Educación Y Cultura, 62, 33-41

Zabalza, M. (2003). Competencias Docentes Del Profesorado Universitario: Calidad Y Desarrollo Profesional. Narcea, 2003. 